\title{
A Discrete Cosine Adaptive Harmonic Wavelet Packet and Its Application to Signal Compression
}

\author{
Nandini Basumallick, S. V. Narasimhan \\ Aerospace Electronics and Systems Division, National Aerospace Laboratories, Council of Scientific and Industrial Research (CSIR), \\ Bangalore, India. \\ Email: narasim@nal.res.in
}

Received October 29 $9^{\text {th }}$, 2010; revised November $13^{\text {th }}$, 2010; accepted November $15^{\text {th }}, 2010$.

\begin{abstract}
A new adaptive Packet algorithm based on Discrete Cosine harmonic wavelet transform (DCHWT), (DCAHWP) has been proposed. This is realized by the Discrete Cosine Harmonic Wavelet transform (DCHTWT) which exploits the good properties of DCT viz., energy compaction (low leakage), frequency resolution and computational simplicity due its real nature, compared to those of DFT and its harmonic wavelet version. Hence the proposed wavelet packet is advantageous both in terms of performance and computational efficiency compared to those of existing DFT harmonic wavelet packet. Further, the new DCAHWP also enjoys the desirable properties of a Harmonic wavelet transform over the time domain WT, viz., built in decimation without any explicit antialiasing filtering and easy interpolation by mere concatenation of different scales in frequency (DCT) domain with out any image rejection filter and with out laborious delay compensation required. Further, the compression by the proposed DCAHWP is much better compared to that by adaptive WP based on Daubechies-2 wavelet (DBAWP). For a compression factor (CF) of 1/8, the ratio of the percentage error energy by proposed DCAHWP to that by DBAWP is about $1 / 8$ and $1 / 5$ for considered $1-D$ signal and speech signal, respectively. Its compression performance is better than that of DCHWT, both for 1-D and 2-D signals. The improvement is more significant for signals with abrupt changes or images with rapid variations (textures). For compression factor of 1/8, the ratio of the percentage error energy by DCAHWP to that by DCHWT, is about $1 / 3$ and $1 / 2$, for the considered 1-D signal and speech signal, respectively. This factor for an image considered is $2 / 3$ and in particular for a textural image it is $1 / 5$.
\end{abstract}

Keywords: Adaptive Harmonic Wavelet Packets, Discrete Cosine Transform, Signal Compression

\section{Introduction}

The wavelet transform (WT) provides a frequency dependent resolution so that the high and low frequencies have a coarse and fine frequency resolution. This is based on the assumption that high and low frequencies require, fine time resolution and coarse frequency resolution; respectively. But to achieve for high frequencies, a finer frequency resolution and for low frequencies, a finer time resolution; the wavelet packet (WP) system is used [1]. It also allows flexibility of selection of wavelet tree structure that enables the WP to select an optimum time-frequency tiling for a given data. This is achieved by adaptive WP and compared to the normal WT, it is more attractive, for applications like signal compression and transient detection [2-5]. This is because the adaptive nature of WP facilitates better energy compaction.

Basically, the WP is a generalization of the WT. In WT, only the coarse/approximation is split at each stage and the detail is carried down as it is. Unlike this, in WP at each stage, the detail/highpass filter branch is also further split and decimated similar to the approximation/ lowpass branch. Thus the complexity of the WP algorithm is more as compared to that of the conventional WT. Hence filterbank realization of WP is cumbersome as it involves additional filtering and sampling rate conversion.

To simplify the adaptive WP, a Harmonic Wavelet Packet based on Discrete Fourier Transform (DFAHWP) 
has been used [4]. This is possible as the Harmonic WT (HWT) due to its built in decimation and interpolation and absence of any explicit antialiasing and image rejection filtering. However for such a HWT, its coefficients not only suffer from leakage effect but also are of complex nature. Further for an image (2D signal), the HWT complex nature involves approximation which results in neglection of the imaginary part of a coefficient once along the rows and columns during each LL stage splitting [9]. These drawbacks are carried over to the DFAHWP also. To overcome these limitations, a HWT based on Discrete Cosine Transform (DCHWT) has been used [8,9]. This is based on the fact that DCT provides real coefficients, which are also less affected by leakage $[8,12]$. The DCHWT has been used for computationally efficient and better quality signal compression and subband spectral estimation [9]. This has also been applied for reducing the cross-term effect, which occurs in Wigner-Ville distribution [10]. Further, a shift invariant version of DCHWT has been considered for applications like signal denoising which reduces glitches in the reconstructed signals [11].

In this paper, an adaptive Harmonic Wavelet Packet based on DCT (DCAHWP) is proposed. The method is computationally simple, as it is based on grouping of real DCT coefficients and the WP decomposition up to the last level is readily available in DCT domain without any repeated filtering and decimation like in a time domain filter bank. Further, the reconstruction also does not involve any explicit interpolation and its associated filtering. The new algorithm has a better performance than the DFAHWP as its scales are less affected by leakage effect and does not involve any approximation for 2D signals. The DCAHWP has been applied both for 1-D and 2-D signal compression and its performance is better compared to that of DCHWT. Further its performance is superior to that of DBAWP for 1D signals.

\section{Adaptive Wavelet Packet}

The WP decomposition for a signal, showing the split of detail/ highpass branch in addition to coarse/lowpass branch, is illustrated in Figure 1(a). For a signal of $N$ points, this generates an array of $M \times N$ coefficients where $M$ is the total number of decomposition stages, given by $M=\log _{2} N$. From this array, $N$ coefficients can be selected to represent the signal. These $N$ coefficients can be that of normal WT decomposition or any other combination.

Wavelet packet facilitates selection of an optimal basis for a given signal. In an adaptive wavelet packet, a specific combination of all the branches is selected to represent a particular signal based on some predefined criterion. The criterion generally aims at having maximum information with minimum possible number of coefficients.

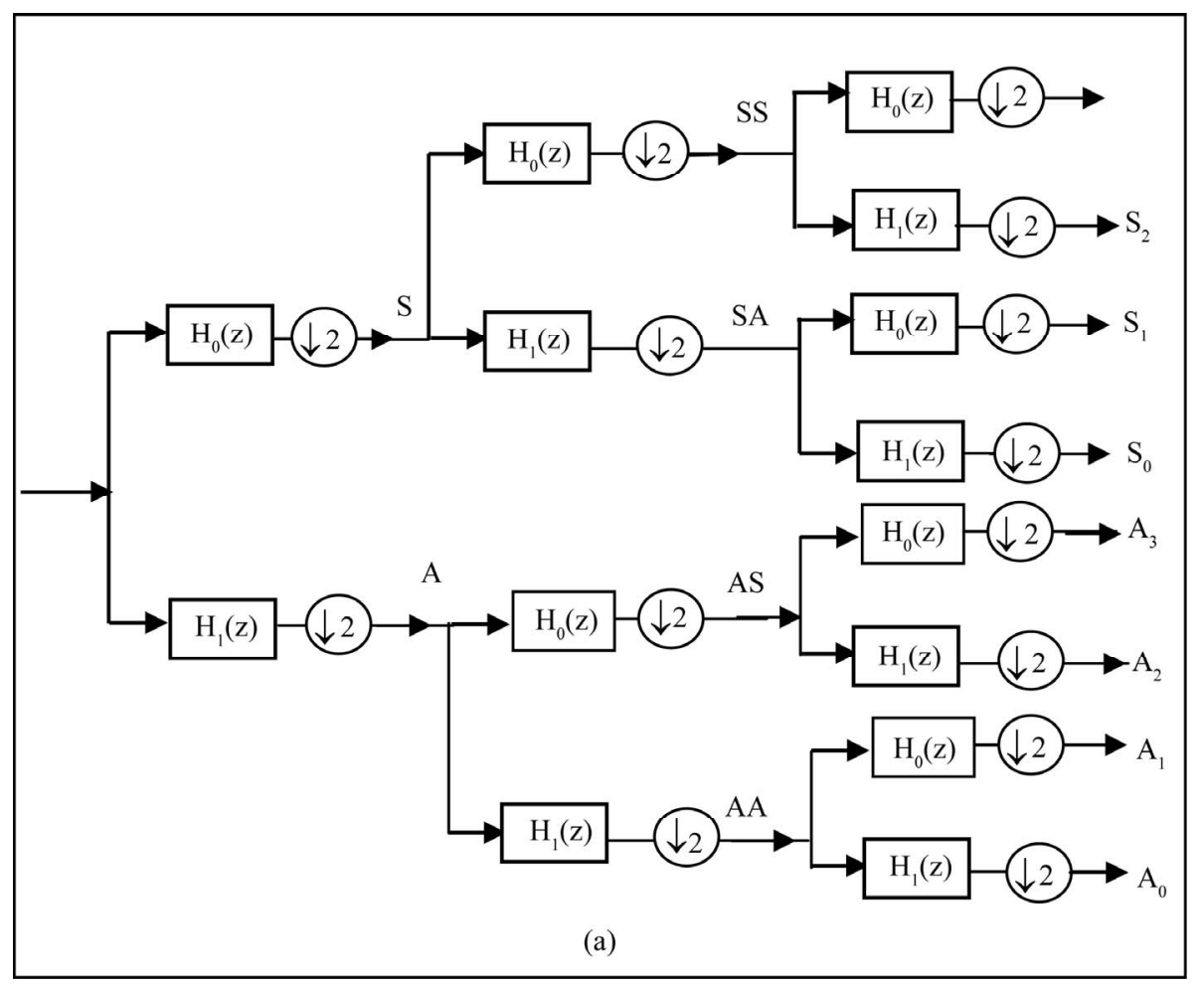




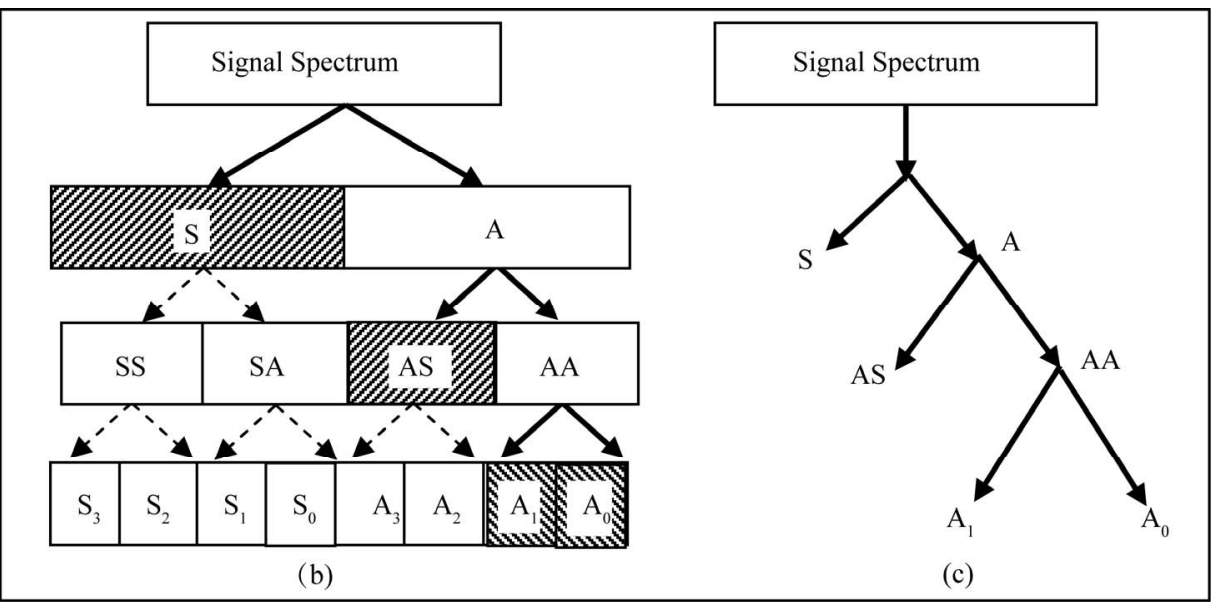

Figure 1. (a) Wavelet packet showing the split of detail/high pass branch in addition to coarse/lowpass branch (b) Optimum scale tree selection, (c) Optimum scale tree.

That is, it minimizes the number of nonzero coef ficients of the resulting wavelet transform. The criterion or cost function used in practice is the Shannon's entropymeasure, given by

$$
E(p)=-\sum_{i=0}^{L-1} p_{i} \log \left(p_{i}\right)
$$

$\left(p_{i} \log \left(p_{i}\right)=0\right.$ if $\left.p_{i}=0\right)$

where $E(p)$ is the entropy for a particular scale, and $L$ is the number of wavelet packet coefficients in that scale. $p_{i}$ are the normalized energies of the wavelet packet coefficients to be considered and are given by [4]

$$
p_{i}=\frac{\left|x_{i}\right|^{2}}{\|s\|^{2}},\|s\|^{2}=\sum_{n=0}^{N-1}|s(n)|^{2}
$$

where, $\left|x_{i}\right|$ are the absolute values of wavelet packet coefficients in that scale, $\|s\|^{2}$ is the normalization factor and $s(n)$ is the original signal of length $N$.

Let $H$ be the Hilbert space, $v \in H$ and $H=\oplus \sum H_{i}$ be an orthogonal decomposition of $H$. The entropy of $v$ relative to the decomposition $H_{i}$ of $H$ is defined $[3,6]$ as a measure of the distance between $v$ and the orthogonal decomposition.

$\varepsilon^{2}\left(v ;\left\{H_{i}\right\}\right)=-\sum\left\|v_{i}\right\|^{2} \ln \left\|v_{i}\right\|^{2}$

$\varepsilon^{2}$ is characterized by the Shannon equation.

If $H=\sum H_{i} \oplus \sum H_{j}=H_{+} \oplus H_{-}, \quad H_{i}$ and $H_{j}$ give the orthogonal decompositions $H_{+}=\sum H_{i}$ and $H_{-}=\sum H_{j}$. Then the Shannon entropy is given by $\varepsilon^{2}\left(v ;\left\{H_{i}, H_{j}\right\}\right)=-\frac{\left\|v_{+}\right\|^{2}}{\|v\|^{2}} \ln \frac{\left\|v_{+}\right\|^{2}}{\left\|v_{i}\right\|^{2}}-\frac{\left\|v_{-}\right\|^{2}}{\|v\|^{2}} \ln \frac{\left\|v_{-}\right\|^{2}}{\|v\|^{2}}+$

$$
\left\|v_{+}\right\|^{2} \varepsilon^{2}\left(\frac{v_{+}}{\left\|v_{+}\right\|} ;\left\{H_{i}\right\}\right)+\left\|v_{-}\right\|^{2} \varepsilon^{2}\left(\frac{v_{-}}{\left\|v_{-}\right\|} ;\left\{H_{j}\right\}\right)
$$

This equation enables searching for the smallest entropy expansion of a signal. The best basis algorithm minimizes the cost function for the transform coefficients. This involves complete decomposition according to the wavelet packet transform.

For the wavelet packet decomposition of a signal shown in Figure 1a, the entropies for different scales are as given below:

The entropy for scale $A_{0}$,

$$
E_{A_{0}}=\sum_{i=0}^{L_{A 0}-1}\left[\frac{\left|x_{A_{0 i}}\right|^{2}}{\|s\|^{2}} \cdot \log \left(\frac{\left|x_{A_{0 i}}\right|^{2}}{\|s\|^{2}}\right)\right]
$$

The entropy for scale $A_{1}$,

$$
E_{A_{1}}=\sum_{j=0}^{L_{A 1}-1}\left[\frac{\left|x_{A_{1} j}\right|^{2}}{\|s\|^{2}} \cdot \log \left(\frac{\left|x_{A_{1} j}\right|^{2}}{\|s\|^{2}}\right)\right],
$$

And the entropy for scale $A A$,

$$
E_{A A}=\sum_{k=0}^{L_{A A}-1}\left[\frac{\left|x_{A A k}\right|^{2}}{\|s\|^{2}} \cdot \log \left(\frac{\left|x_{A A k}\right|^{2}}{\|s\|^{2}}\right)\right] \text {. }
$$

Here $x_{A_{0} i}, x_{A_{1} j}$ and $x_{A A k}$ are the wavelet packet coefficients in the scales $A_{0}, A_{1}$ and $A A$, having $L_{A 0}$, $L_{A 1}$ and $L_{A A}$ samples; respectively. The entropies of other scales can also be found in a similar manner.

The entropy will be low when energy is concentrated only at few locations, further it will be zero only when all the energy of the signal is at one coefficient. On the other 
hand, the entropy will be maximum when energy is equally distributed over all coefficients. Hence the set of $N$ wavelet packet coefficients for which entropy is least provides the efficient representation of the signal. This enables to select those coefficients set which has maximum concentration of the energy.

To choose such a set, at each scale, each pair of partitioned coefficient sets is compared with those of their parent from which the partitions are derived. If the combined partition coefficients have entropy smaller than that of their parent, the partitions are considered, otherwise the parent.To make further comparisons while proceeding upwards (towards parent), if partitions are considered, then their entropy is assigned to their parent. In this way the complete wave packet array is scanned and this provides an optimal time-frequency tiling in terms of location of coefficient energy. The Figure 1b shows the method of optimum splitting for an arbitrary case. Here, the combined entropy of $S$ and $A$ is less than the signal spectrum entropy. Hence, this signal spectrum splitting into $S$ and $A$ is valid. Further for $S$, its splitting into $S S$ and $S A$ is not valid as the combined entropy of $S S$ and $S A$ is greater than that of $S$. But splitting of $A$ into $A S$ and $A A$ is valid as their combined entropy is less than that of $A$. Further, split of AS into $A_{3}$ and $A_{2}$ is not valid as their combined entropy is larger than that of $A S$. But $A A$ split into $A_{1}$ and $A_{0}$ is valid as their combined entropy is less than that of $A A$. The optimum scale tree selected is shown in the Figure 1c.

The decimation of the scales in the scale tree selected depends up on the bandwidth of the individual scales resulting in a time-frequency tiling and this may totally differ from the normal dyadic tiling. Such a tiling results in a maximum energy compaction for a given number of coefficients. It is not only the time frequency tiling but also the wavelet function used, also determines the performance of compression. That is, a good wavelet for a particular signal results in a good energy compaction with least number of coefficients. Thus the wavelet packet can result in an efficient lossless compression. This type of scale tree selection is also known as best basis selection.

\section{Discrete Cosine Harmonic Wavelet Transform}

The filter bank realization of WT, involves decimation of the scale components. The restoration of the processed overall spectrum corresponding to the original sampling rate, involves interpolation and summation of the interpolated scale outputs in time. The harmonic wavelet transform based on DFT (DFHWT) realizes the subband decomposition in the frequency domain by grouping the Fourier transform (FT) coefficients and the inverse of these groups results in decimated signals [7]. Further after processing, the FT of the scales can be repositioned in their corresponding positions to recover the overall spectrum, with the original sampling rate. Therefore, this will not involve explicit decimation and interpolation operations. As a consequence, no band limiting and image rejection filters are necessary. Also, while reconstruction, there are no delay compensations as the scales are combined in the frequency domain by repositioning them. In view of this, the harmonic subband decomposition is very attractive due to its simplicity. Further, the decomposition being done in frequency domain, it is well suited for those processing methods which are performed in frequency domain, like group delay processing [8].

For a 1-D signal in the DFHWT, the grouping of the DFT coefficients with possible conjugate symmetry though makes the WT coefficients complex; this will not pose any problem for reconstruction as after concatenation of the groups, the conjugate symmetry is restored to get the real signal.

The DFHWT is very attractive as long as no processing of the components is involved prior to inverse transformation. However, for a signal segment obtained without using any window function, there can be a severe leakage effect from one scale to another scale. If different scales have to be processed differently, this is not achieved as the signal energy from one to another has already leaked. The DFHWT may be tolerable for a signal with well-separated frequency components of sufficiently high magnitude. But for closely spaced components of significantly different magnitudes, during the computation of the FT itself, the energy will leak from the higher amplitude component to the lower one (and vice versa). This results in a large bias in the spectral magnitude and may even totally eclipse smaller amplitude spectral peaks. In such a case, decomposing the signal based on DFHWT and processing the subbands may not be very effective. Further leakage in DFHWT will also limit its use in signal or image compression application. The reason for this is that it is not possible to get a good signal reconstruction by omitting the lower scales (corresponding to high frequencies) in WT as the leaked energy cannot be recovered unless all the scales are considered

Therefore to utilize the attractive features of the harmonic wavelet transform, DCT is used instead of DFT, which has a comparatively reduced leakage effect. This is due to symmetrical data extension which results in a smooth transition from one DCT period to the other without any discontinuity [8].

The wavelet transform $W_{x}(a, b)$ characterizes the cor- 
relation or similarity between the signal $x(t)$ to be analyzed and the wavelet function $\psi((t-b) / a)$. Such a correlation is given by

$$
W_{x}(a, b)=\frac{1}{a^{1 / 2}} \int_{-\infty}^{\infty} x(t) \psi^{*}\left(\frac{t-b}{a}\right) d t
$$

where $\psi(t)$ is the prototype/mother wavelet. By shifting and scaling $\psi(t)$ by the parameters $b$ and $a$, respectively; all the basis functions $\psi_{a, b}(t)=a^{-1 / 2} \psi((t-b) / a)$ are obtained. Eqn. (3) can be realized in the frequency domain using Parseval's theorem as [13]

$$
W_{x}(a, b)=\frac{a^{1 / 2}}{2 \pi} \int_{-\infty}^{\infty} X(\omega) \Psi^{*}(a \omega) e^{j \omega b} d \omega
$$

Therefore, the wavelet transform can be derived by windowing the spectrum $X(\omega)$ with $\Psi^{*}(a \omega)$ and inverse Fourier transforming the product.

$$
W_{x}(a, b)=a^{1 / 2} F^{-1}\left[X(\omega) \Psi^{*}(a \omega)\right]
$$

$\Psi(\omega)$ and $X(\omega)$ are the FT of the mother wavelet $\psi(t)$ and the signal $x(t)$. That is, $W_{x}(a, b)$ for a particular scale ' $a$ ' can be computed by the Eqn. (4b) using $X(\omega)$ and $\Psi(a \omega)$ by FFT algorithm.

For a real symmetric signal $x_{s}(t)$ and a real symmetric wavelet $\psi_{s}(t)$ function, Eqn.(2a) becomes [9]

$$
C_{x}(a, b)=\frac{a^{1 / 2}}{2 \pi} \int_{-\infty}^{\infty} X_{s}(\omega) \Psi_{s}(a \omega) \cos (\omega b) d \omega
$$

$X_{s}(\omega)$ and $\Psi_{s}(\omega)$ are the Fourier transform of $x_{s}(t)$ and $\psi_{s}(t)$ respectively. (Generally the wavelet function is a symmetrical one but to have consistency in the notation $\psi_{s}(t)$ is used). In other words, they are the cosine transforms of $x_{s}(t)$ and the mother wavelet $\psi_{s}(t)$. $C_{x}(a, b)$ is the wavelet transform in cosine domain instead of Fourier domain. Hence the corresponding equation for Eqn.(4b) is

$$
C_{x}(a, b)=a^{1 / 2} C^{-1}\left[X_{s}(\omega) \Psi_{s}(a \omega)\right]
$$

Therefore the cosine wavelet transform coefficient $C_{x}(a, b)$ for a particular scale ' $a$ ' can be computed by the Eqn. (5b) using $X_{s}(\omega)$ and $\Psi_{s}(a \omega)$ by a fast cosine transform algorithm which indirectly uses FFT algorithm. $\Psi_{s}(\omega)$ is very simple for the Harmonic cosine wavelet transform (CHWT), and it is zero at all frequencies except constant over a small frequency band.

$$
\Psi_{s}(\omega)=\left\{\begin{array}{l}
1, \quad \omega_{0}-\omega_{c}<\omega<\omega_{0}+\omega_{c}, \\
-\omega_{0}-\omega_{c}<\omega<-\omega_{0}+\omega_{c}, \\
0, \text { otherwise }
\end{array}\right.
$$

For this the wavelet $\psi_{s}(t)$ is,

$$
\begin{aligned}
& \psi_{s}(t)=\frac{\omega_{c}}{\pi} \frac{\sin \omega_{c} t}{\omega_{c} t} \cos \omega_{0} t \\
& \text { Representing } \frac{\sin \omega_{c} t}{\omega_{c} t} \text { by } \sin c\left(\omega_{c} t\right), \\
& \psi_{s}(t)=\frac{\omega_{c}}{\pi} \cos \omega_{0} t \sin c\left(\omega_{c} t\right)
\end{aligned}
$$

Hence the mother wavelet is a cosine modulated sinc function. Here the decomposition of the signal in the frequency domain is simple but suffers from the problem of poor time localization due to slow decaying of the sinc function. Though a spectral weighing other than rectangular improves the localization in time it results in a non-orthogonal wavelet set. The type of spectral weighing will determine the wavelet as it is the cosine transform of the wavelet.

For the cosine harmonic wavelet transform, the spectral weighing is a symmetrical rectangular function and for a discrete signal it is zero except over symmetrical finite bands $[\pi / p, \pi / q]$ and $[-\pi / p,-\pi / q]$ where $p, q$ can be real numbers, not necessarily integers.

For an orthogonal CHWT, the wavelet function is fixed and corresponds to a rectangular weighing in the frequency domain which results in such a wavelet transform.

The Discrete cosine transform (DCT) enables the implementation of the above cosine transform discussed as it forms the symmetric signals $x_{s}(t)$ and $\psi_{s}(t)$ by itself (for the given non-symmetric $x(t)$ and $\psi(t)$. For a sampled signal $x(n), n=0,1,2, \cdots(N-1)$, the DCT of $N$ points, is defined as the DFT of a $2 N$ point symmetrically extended signal $y(n)$.

$y(n)=\left\{\begin{array}{lc}x(n), & 0 \leq n \leq N-1 \\ x(2 N-1-n), & N \leq n \leq 2 N-1\end{array}\right.$

$y(n)$ is even symmetric with respect to the point $[N-(1 / 2)]$. This leads to DCT and is given by

$$
C_{x}(k)=\left\{\begin{array}{l}
\sum_{n=0}^{N-1} 2 x(n) \cos \frac{\pi k(2 n+1)}{2 N}, 0 \leq k \leq N-1 \\
-C_{x}(2 N-k), N \leq k \leq 2 N-1
\end{array}\right.
$$

Using the above $\Psi_{s}(\omega)$ in the CHWT, the subband decomposition is done in frequency domain unlike in time domain by a filter bank. This is achieved by grouping the $2 N$ coefficients of a discrete cosine transform (DCT) of length $2 N$ and this is equivalent to applying a window or weighing by a constant in the frequency domain.

The DCT coefficients can be grouped in a way similar to that of DFT coefficients and the DCT being real, there 
is no necessity to do the conjugate operation in placing the coefficients symmetrically. The symmetrical placement is also not necessary due to the very definition of the DCT as it provides only half the number of coefficients and the inverse DCT definition takes care of the symmetry. The grouped coefficients for each band have to be treated as if they are the DCT coefficients of that subband Figure 2a.

For the reconstruction, the DCTs of the subband signals are concatenated to get the DCT of the fullband signal. For the first stage of inverse DCHWT illustrated in Figure 2a, the DCTs of the subband signals corresponding to groups $\mathrm{C}_{3}$ and $\mathrm{C}_{4}$ are concatenated. The resulting group of coefficients is concatenated with the DCT of subband signal corresponding to group $\mathrm{C}_{2}$, in the next stage. Again, the resulting group of coefficients is concatenated with the DCT of subband signal corresponding to group $\mathrm{C}_{1}$, to form the DCT of the fullband signal.

The 1-D DFHWT or DCHWT can be extended to 2-D signals (images) by applying it to rows and columns separately. For images, the DFHWT performance is poor, even considering 100\% coefficient (without omitting any coefficient). This is due to the approximation in the algo rithm which takes into account only the real part of a complex coefficient, both during row and column wise coefficient grouping which repeats for every scale.

The DCHWT does not pose such a problem for a 2D signal. This is due to the fact that the DCT is a real transform and the grouping does not involve conjugate symmetry to get real signals. Here for the image, the DCT coefficients of each column are grouped and their inverse DCT results in DCHWT for that column. For each scale (along columns), the DCT coefficients along each row are taken and grouped. The inverse DCT of these groups will result in 2D DCHWT. This procedure is repeated for further scales considering the low-low (LL) subband as input Figure 2b. Considering from one LL to next LL as one stage, it is essential that the LL

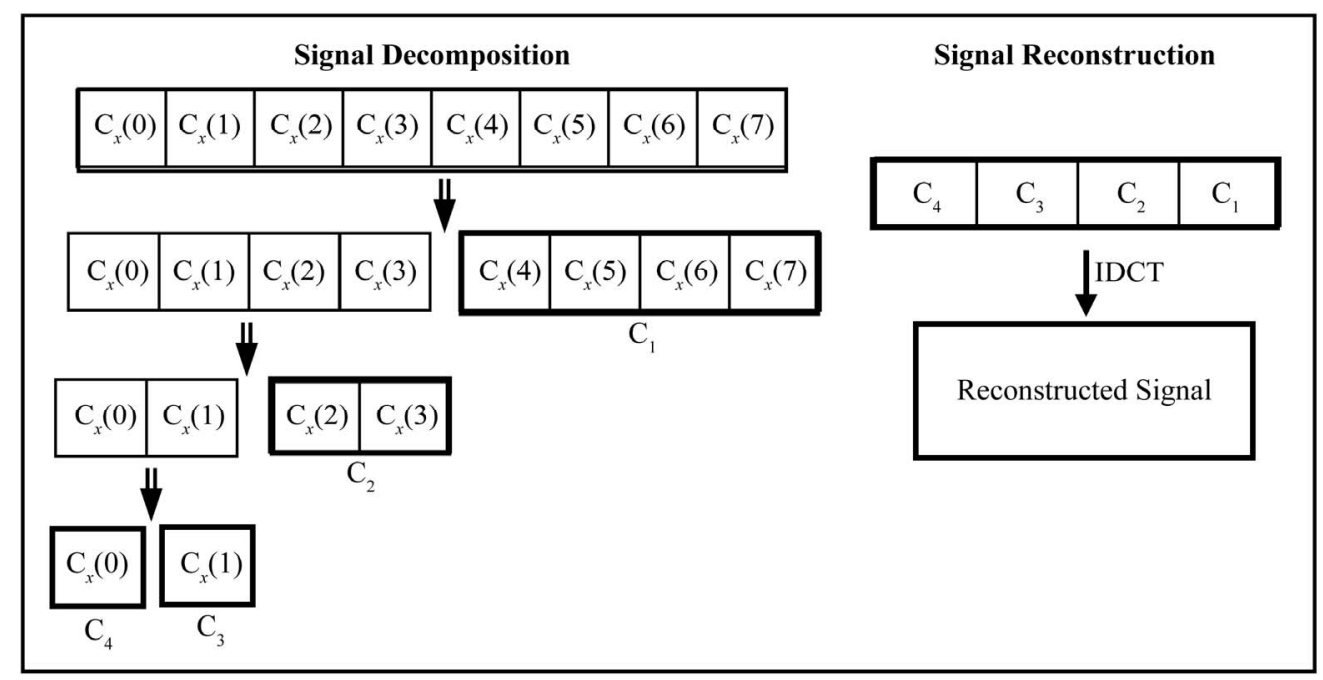

(a)

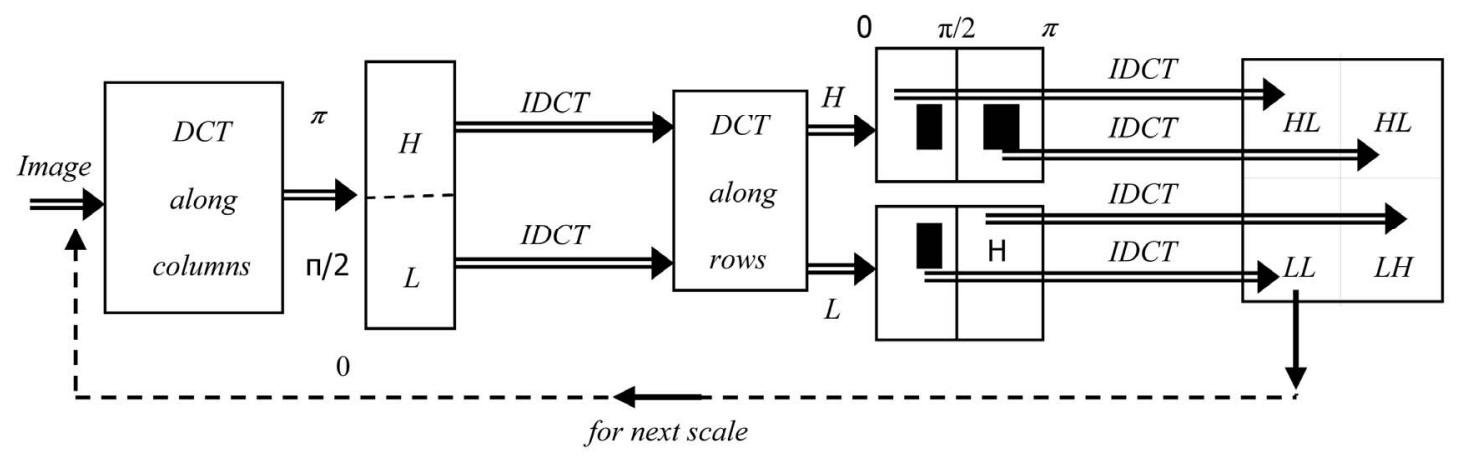

(b)

Figure 2. (a) DCHWT for a 1-D signal, $N=16$, Subbands: $C_{4}, C_{3}, C_{2}, C_{1}$, (b) DCHWT for a 2-D signal. 
subband which goes as input should be in data domain and not in frequency (DCT) domain. This is essential as it enables to split DCT of columns in to $\mathrm{H}$ and $\mathrm{L}$ and proceed further. Since no approximations are made, there will not be any error when the image is constructed with $100 \%$ coefficients [9] which is not so with DFHWT.

\section{Discrete Cosine Adaptive Harmonic Wavelet Packet (DCAHWP)}

The DCAHWP for both 1-D and 2-D signals will be considered in this section.

\subsection{For 1-D Signal}

Adaptive wavelet packets can be realized in the frequency domain using the approach of grouping of frequency domain coefficients (harmonic wavelet packet) [4]. The DCT based harmonic wavelet packet is simpler because the DCT coefficients are nothing but decomposition up to the last possible level and they are directly available without any filtering unlike the discrete wavelet transform realized by a filter bank. Further compared to DFAHWP, their low leakage effect and real nature contribute to the quality and simplicity of DCAHWP. The DCAHWP decomposition is illustrated in Figure3.

Unlike in DCHWT Figure2a, the high subband is also split into two subbands at each decomposition level, apart from the low subband like in any WP. Even though the decomposition up to the last level, to apply the treatment of adaptive WP, it is indicated in Figure $\mathbf{3}$ as if all the DCT coefficients are together and its splitting takes place as in time domain filter bank approach. Here rather levels 2 and 1 are formed by combining the coefficients available at level-3.

Maintaining the total number of coefficients equal to the signal length, different combinations of subbands may be possible for reconstruction. For DCAHWP also, the scales/subbands to be used for reconstruction are selected such that their combined entropy is minimum, as described in Section-2. Here a detailed algorithm for this is given.

Algorithm for DAHWP implementation:

1) Compute the $N$-point Discrete cosine transform (DCT) of the $N$-point signal.

2) The DCT coefficients correspond to the level (last level) $L=\log _{2} N$. Obtain the other $(L-1)$ decompositions of WP by grouping the DCT coefficients. The number of scales/subbands at level $l$ is $2^{l}$ and each is of length $2^{L-l}$ points; $l=1$ to $L$.

3) Starting from the last level, the sum of entropy levels of two adjacent subbands/scales (children) in that level, are compared to the entropy of the band in the previous level (parent) from which they were formed (actually the parent is formed from children here !). If the combined entropy of the children is found to be less than that of the parent, the decomposition is valid. Each time the children are selected, the combined entropy of the children is assigned to their parent for subsequent comparisons.

4) The selected scales/subbands at different levels are used to reconstruct the signal, and the total number of coefficients is $N$. For compression, the number of coefficients to be considered is decided by the compression factor (CF). The selection of these coefficients is based on their magnitude values starting from the largest [2].

\section{For 2-D signal:}

The DCAHWP implementation for a 2-D signal (image) is an extension of the above method. At each decomposition level, each of the subband images (LL, HL, LH and $\mathrm{HH}$ achieved by the block D in Figure 4) is further divided into four equal subbands. This is repeated till the last possible decomposition level, that is when each subband consists of only one pixel Figure 4. For reconstruction, the

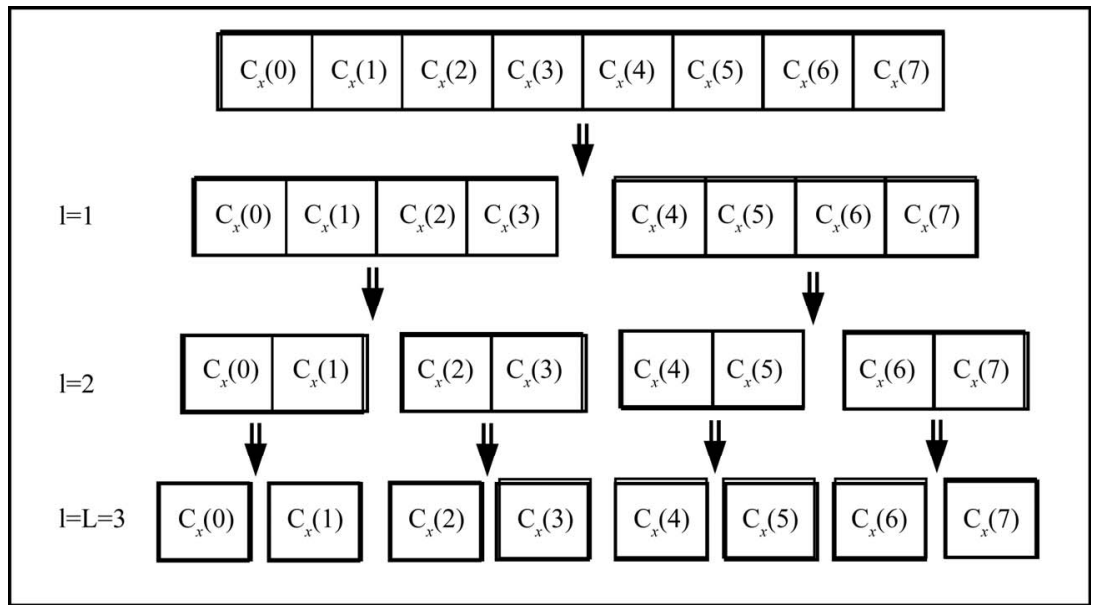

Figure 3. DCT harmonic wavelet packet. 


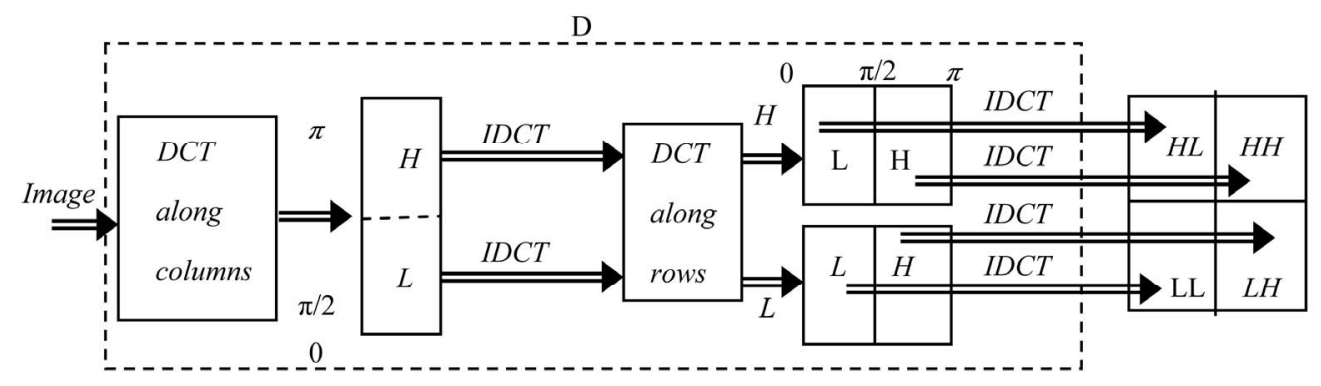

(a)

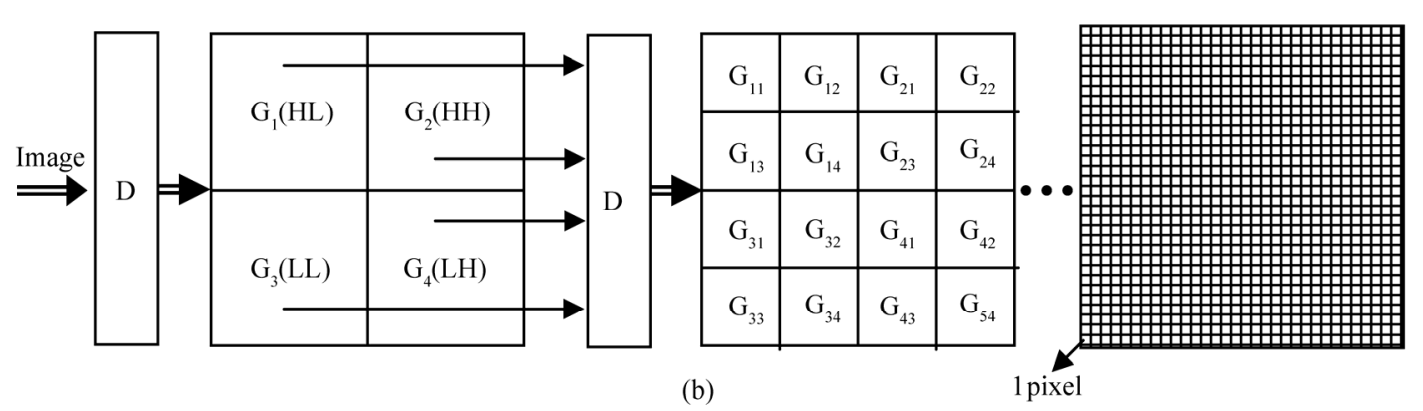

Figure 4. (a) Decomposition of an image into four subbands by DCHWT. (b) DCT Harmonic Wavelet Packet Decomposition for an image.

combined entropy of four children is compared to that of the parent at each level and a combination with lowest entropy is selected. The detailed algorithm is given.

Algorithm for DCAHWP implementation:

1) First take the DCT along columns and then along rows, i.e. compute the 2-D DCT of the $N \times N$ pixel image.

2) Perform the WP decomposition up to the last possible level, $L \quad\left(L=\log _{2} N\right)$. The number of scales/subbands at level $l$ is $4^{l}$ and each is of size $2^{L-l} \times 2^{L-l}$ pixels; $l=1$ to $L$. The decomposition is carried out by grouping the DCT coefficients.

3) Starting from the last level, the entropy comparisons are made between the children and their parent and the scales/subband decomposition tree with the lowest entropy is selected.

4) For reconstructing the image, the selected scales / subbands with a total of $N \times N$ coefficients, are used. For compression a specified number of largest coefficients amongst the $N \times N$ coefficients across the scales are selected. This specific number is decided by the CF.

\section{Simulation Results}

To illustrate the performance of the proposed DCAHWP adaptive wavelet packet, four examples: 1)1-D signal, 2) a speech signal, 3) a 2-D image (512 x 512) and 4) a textural image (64 x 64); are considered. Its performance is compared with that of DCHWT. For the examples 1 and
2, the performance of the proposed method is compared with those of Daubechies-2 based WT (DBWT) and its adaptive WP (DBAWP). For all examples, the performance of proposed WP method is compared with that of DCHWT. Since the performance of DFHWT is inferior to that of DCHWT [7], the proposed DCAHWP has not been compared with those of DFAHWP, the WP based on DFT. The compression performance is evaluated by the index,

$\%$ error energy $=($ error energy $/$ signal energy $) 100$

Example 1 [2]: A signal of 64 samples, having a sinusoid and a spike Figure 5, is decomposed into subbands using both DCHWT and DCAHWP. The signal was reconstructed with and without compression in both the cases.

The decomposition tree structures for DCHWT and DCAHWP are depicted in Figures 6(a), 6(b) respectively.

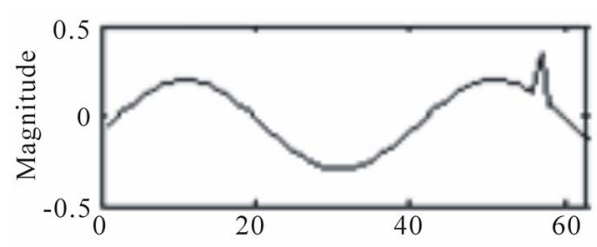

Figure 5. 1-D signal having a sinusoid and a spike. 


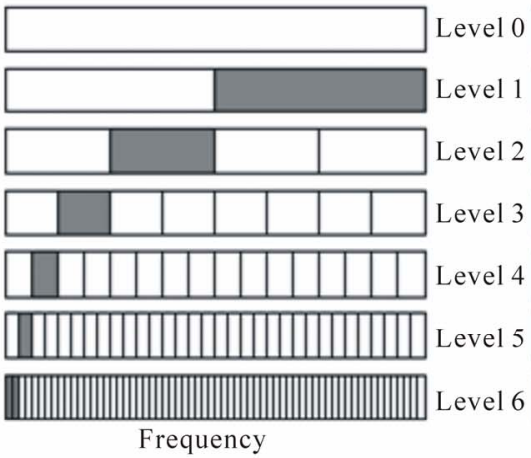

(a)

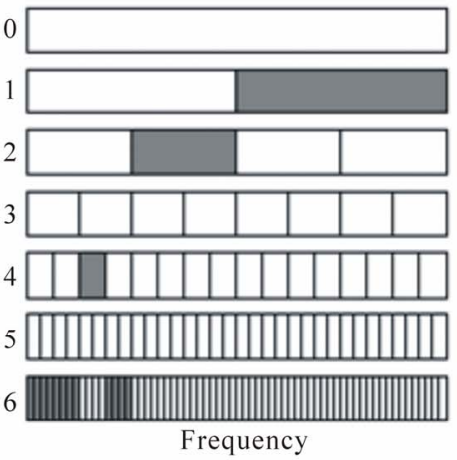

(b)

Figure 6. Decomposition tree for the signal in Figure 5 by: (a) DCHWT (b) DCAHWP.

The frequency band splitting in each decomposition level is indicated by vertical lines. The selected groups are represented by shaded blocks. In DCHWT, the high subband is selected at each level and in the last level the low subband is also selected Figure 6a. But the tree structure for DCAHWP Figure 6b differs from that of DCHWT and depends on the signal characteristics

The DCHWT time-frequency tiling is such that lower scales have larger frequency spacing and smaller time spacing, whereas higher scales have smaller frequency spacing and larger time spacing Figure 7a. But the DCAHWP time-frequency tiling Figure $\mathbf{7 b}$ is according to the best basis selected for the signal on hand. In the Figures $\mathbf{7 a}, \mathbf{7 b}$, larger coefficients are represented by darker shades.

The WP coefficient values at various decomposition levels are shown in Figure 8a. At each decomposition level, each subband is further split into two subbands. For example for the level 2, Figure 8a shows the WP coefficients for the four scales; the different scales are put side by side. The same is valid for other levels also. The selected coefficients for DCHWT and DCAHWP are shown in Figures. 8b, 8c respectively. For DCHWT, a fixed set of the coefficients is selected; whereas for DCAHWP, the set of coefficients varies according to the signal. For DCAHWP the frequency band splitting is denser, providing higher frequency resolution in the low frequency range (Figure $\mathbf{8 b}$ ). This is also found in the time-frequency plot (Figure $\mathbf{7 b}$ ) and the tree structure (Figure 6b) for DCAHWP. However, this is not so for DCHWT (Figures. 6a, 7a, 8a). For compression, only 8 largest coefficients from the selected 64 Figure 8(b, c), are used to reconstruct the signal.

Signal compression performance of DCAHWP was compared to that of DCHWT and also with that of DBWT and DBAWP. The reconstructed signal by DCAHWP with $C F=1 / 8$, contains a well defined spike in the same position as in the original (Figure 9d). But for the same CF, the spike is not clear in the reconstructed signal by DCHWT (Figure 9c). The performance of DBAWP is poor and that of DBWT is even poorer, as neither the spike nor the sinusoid are clearly brought out (Figure. 9a, b). The error plots corresponding to Figure 9(a-d) are shown in Figure 9(e-h) and it is seen that the magnitude of the error for compression by DCAHWP is significantly less than that by other methods. Further, the percentage error energy by DCAHWP is about $1 / 3^{\text {rd }}$ of that by DCHWT and about $1 / 8^{\text {th }}$ that of DBAWP Table 1.

\section{Example 2:}

For a speech signal (Figure 10) also, the results obtained are similar to those for example-1. For the compression factor $1 / 8$, the performance of DBWT and of DBAWP are inferior compared to that of DCHWT. Between DBAWP and DBWT, the former is better as is optimal for the Daubechies-2 wavelet. But DCAHWP resulted in best performance compression compared to the other methods, which is clear from comparing the reconstruction errors for the various methods (Figure 11e-h). The percentage error energy by DCAHWP is about $1 / 5^{\text {th }}$ of that by DBAWP and about $1 / 2$ of that by DCHWT Table 2.

Table 1. \% error energy (\% EE) for reconstruction of the signal in Fig 5 with $1 / 8$ coefficients $(C F=1 / 8)$.

\begin{tabular}{cc}
\hline Method & \% EE with CF $=1 / 8$ \\
\hline DBWT & 13.4947 \\
DBAWP & 5.8149 \\
DCHWT & 1.9550 \\
DCAHWP & 0.7396 \\
\hline
\end{tabular}




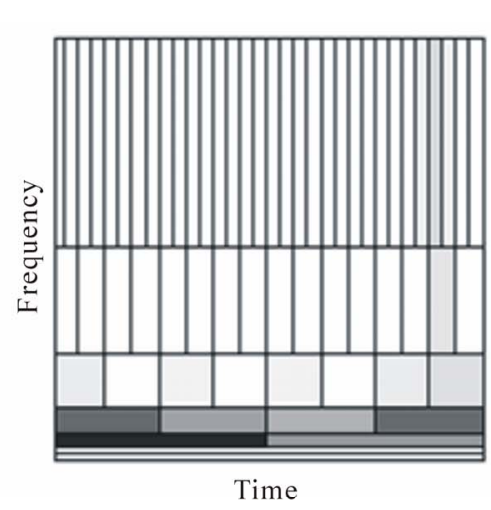

(a)

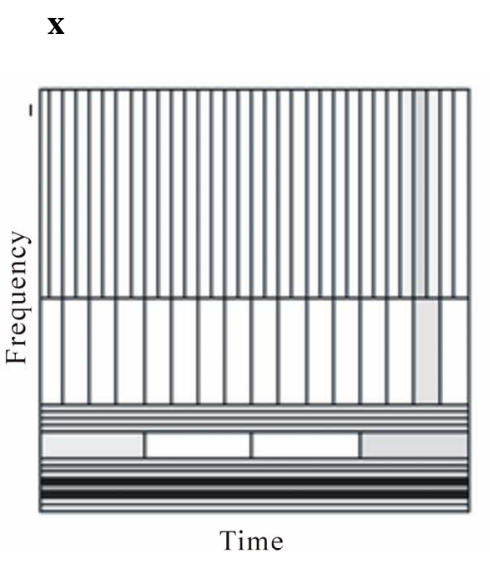

(b)

igure 7. Time-frequency tiling for the signal in Figure 5 by: (a) DCHWT (b) DCAHWP.

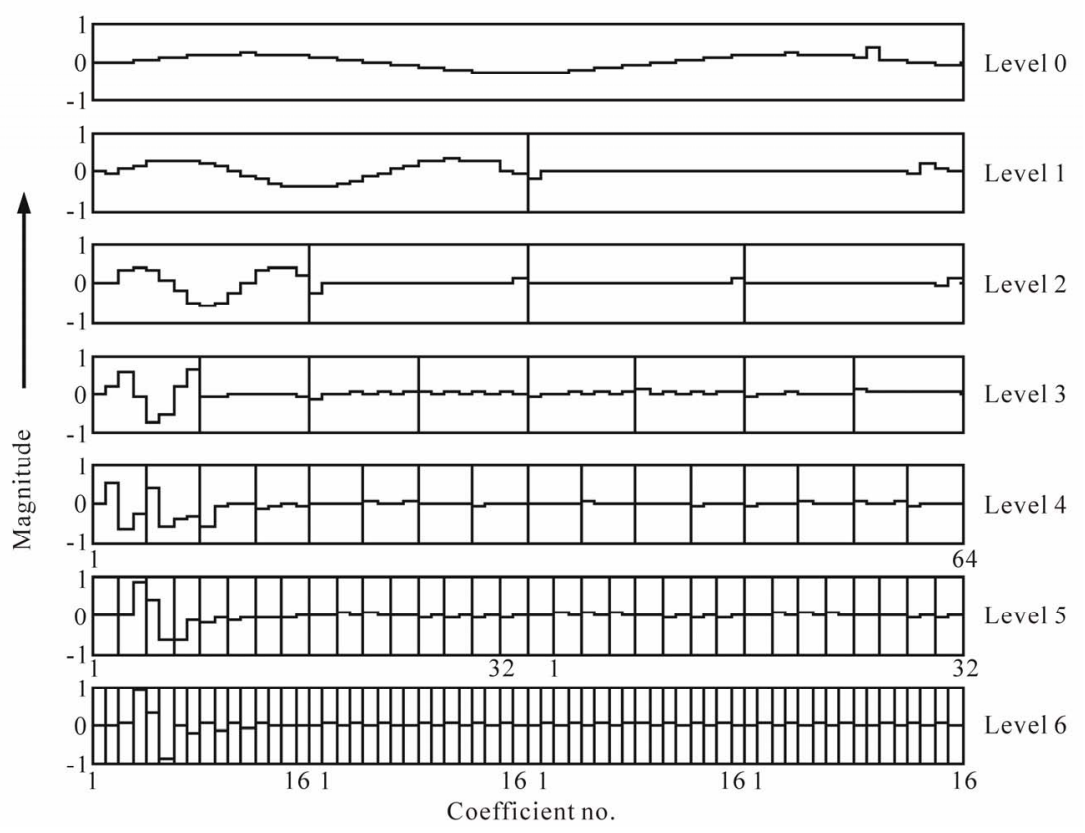

Figure 8. WP decomposition coefficients for the signal in Figure 5.

Table 2. \% error energy (\% EE) for reconstruction of the speech in Figure 10 with 1/8 coefficients (CF= 1/8).

\begin{tabular}{cc}
\hline Method & \% EE with CF $=1 / 8$ \\
\hline DBWT & 4.2695 \\
DBAWP & 2.7425 \\
DCHWT & 1.1542 \\
DCAHWP & 0.5886 \\
\hline
\end{tabular}

Example - 3: The performances of DCAHWP and DCHWT were compared for a $512 \times 512$ 'Barbara' image. The compression results for a CF of $1 / 32$ clearly
Indicate the superior performance of DCAHWP over DCHWT (Figure 12b, 12c). There is a significant blurring in the reconstructed image by DCHWT and hence the edges like eyes, nose and stripes are not distinct Figure 12c. In contrast, the edge information is preserved to a large extent in reconstructed image by DCAHWP, which is manifested as the sharpness of the nose, eyes and stripes Figure 12c.

The above results are supported by the percentage error energy index as for DCAHWP, it is only about $2 / 3^{\text {rd }}$ that of DCHWT Table 3.

Example 4: To illustrate the performance superiority of DCAHWP due to its best basis selection over DCHWT, 

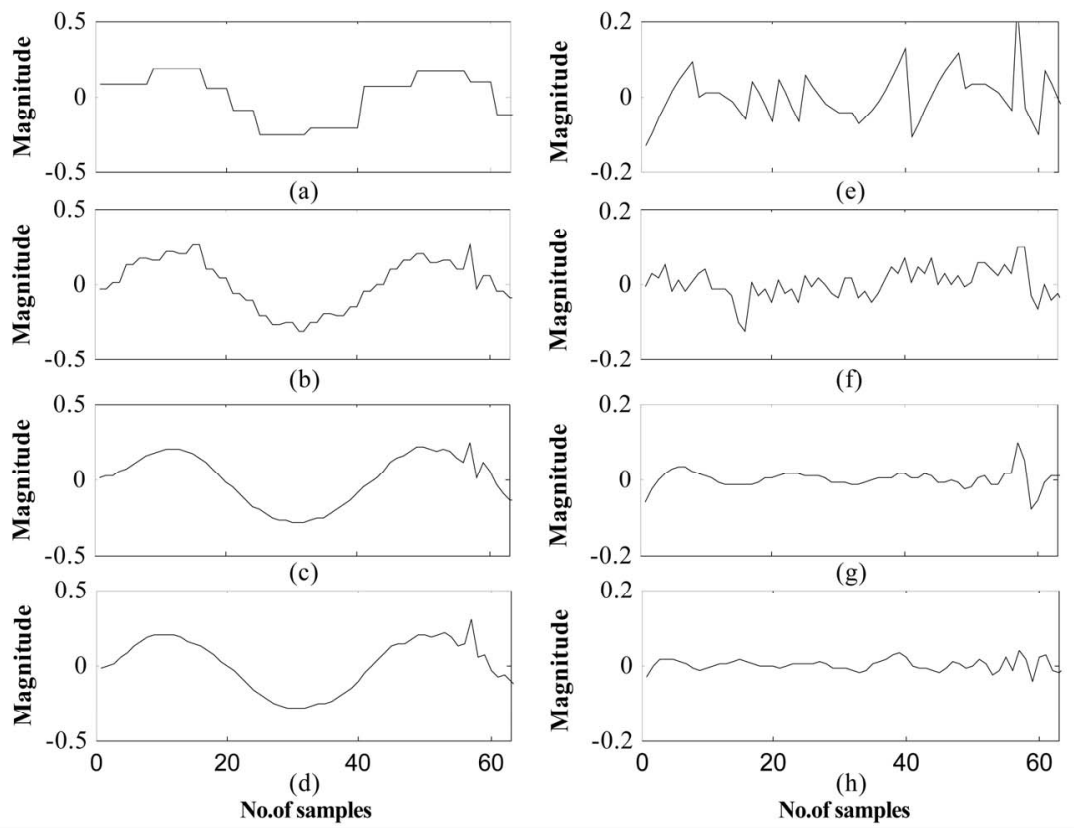

Figure 9. Reconstruction of the signal in Figure 5 a. (a-d), Reconstructed signal for CF=1/8 by, (a) DBWT, (b) DBAWP, (c) DCHWT, (d) DCAHWP (e-h), error corresponding to (a-d).

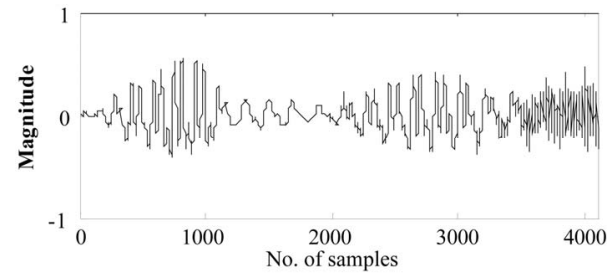

Figure 10. Speech signal.
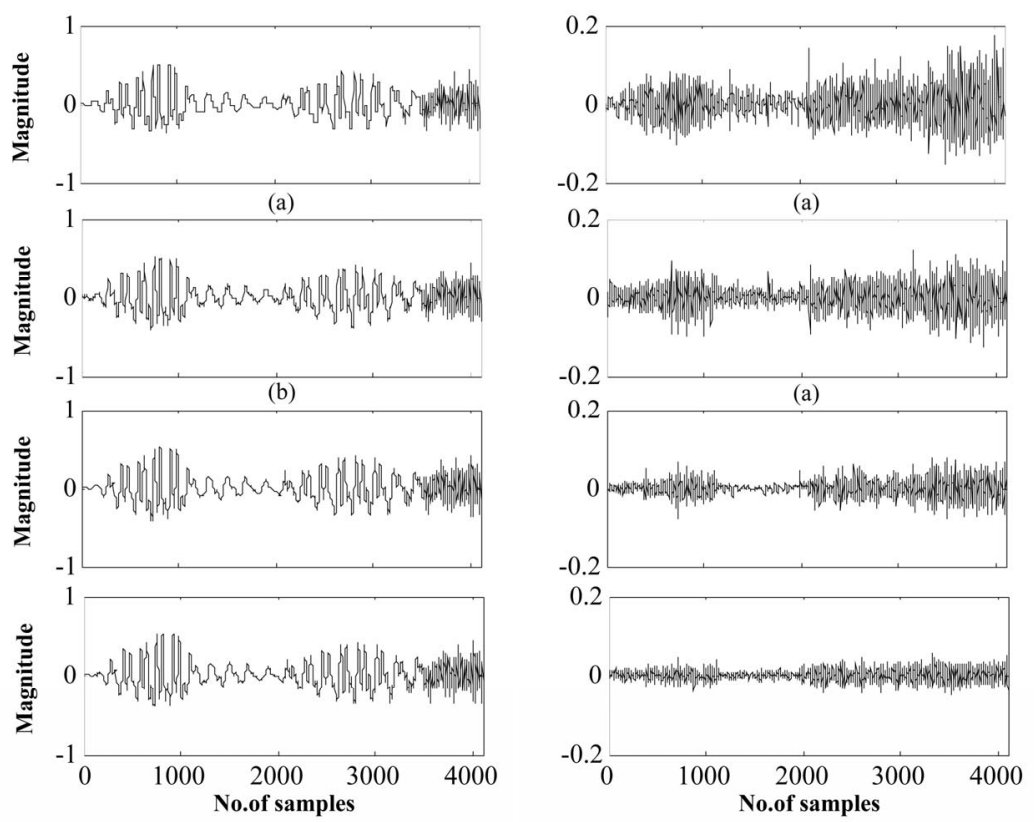

Figure 11. (a-d): Reconstructed speech for CF=1/8 by (a) DBWT (b) DBAWP, (c) DCHWT, (d) DCAHWP , (e-h): error corresponding to (a-d). 


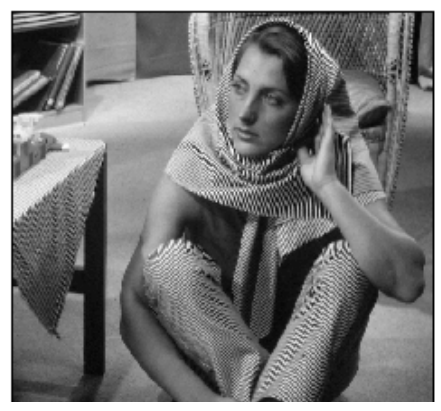

(a)

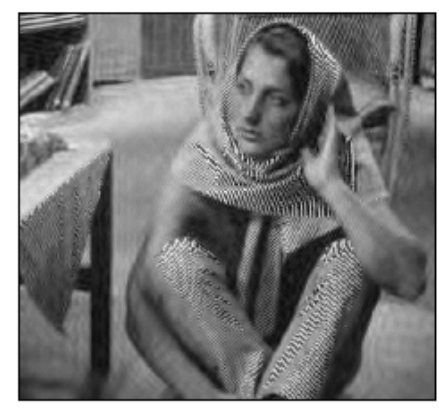

(b)

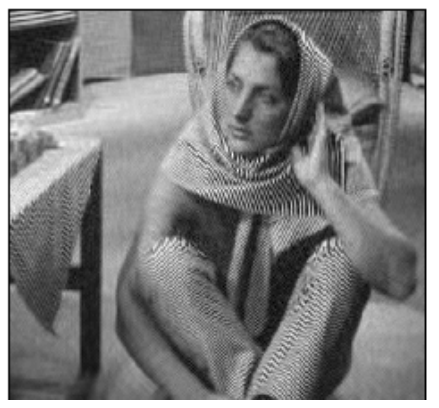

(c)

Figure 12 Image reconstruction of the 'Barbara' image (512×512), (a) Original, (b) DCHWT (CF=1/32), (c) DCAHWP $(\mathrm{CF}=1 / 32)$.

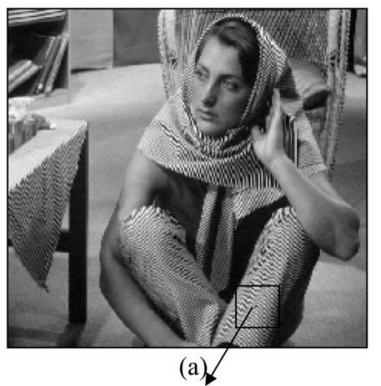

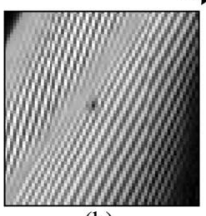

(b)

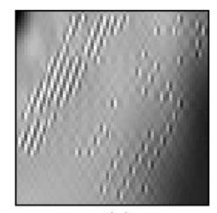

(c)

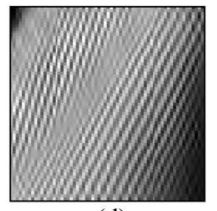

(d)

Figure 12. Image reconstruction of the 'Barbara' image $(512 \times 512)$, a) Original Barbara image, (b) Part of original with in the square(64×64), (c) DCHWT (CF=1/32), (d) DCAHWP (CF=1/32).

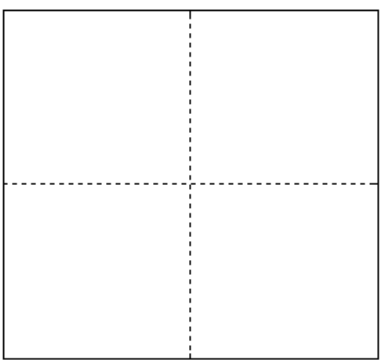

(a)

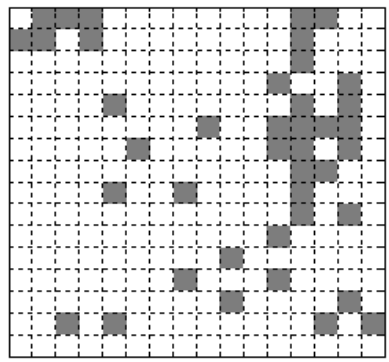

(d)

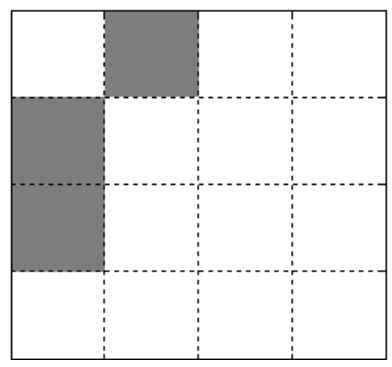

(b)

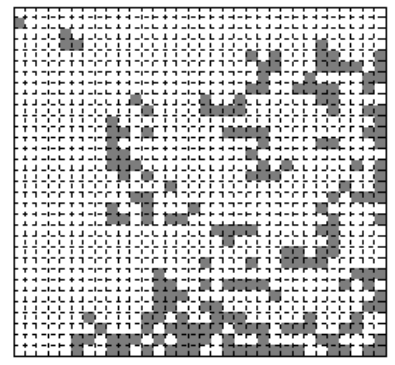

(e)

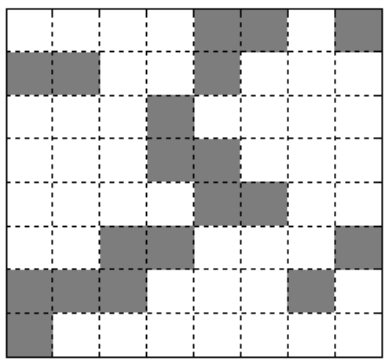

(c)

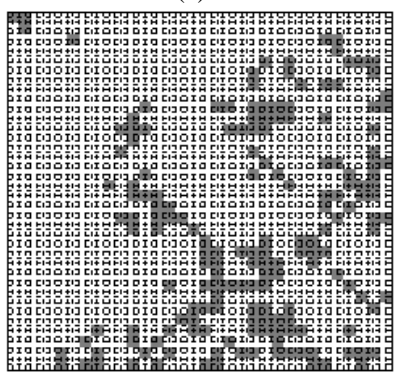

(f)

Figure 14 Adaptive wavelet packet decomposition tree for the image in Figure 13a. (a-f): Decomposition levels 1-6 respectively. Shaded blocks indicate selected wavelet coefficients at each decomposition level. 
Table 3. \% error energy (\% EE) for reconstruction of the image in Figure 12a with 1/32 coefficients $(C F=1 / 32)$.

\begin{tabular}{cc}
\hline Method & \% EE with CF $=1 / 32$ \\
\hline DCHWT & 1.1310 \\
DCAHWP & 0.7517 \\
\hline
\end{tabular}

Table 4. \% error energy (\% EE) for reconstruction of the image in Figure 13(a) with $1 / 32$ coefficients $(C F=1 / 32)$.

\begin{tabular}{cc}
\hline Method & \% EE with CF $=1 / 32$ \\
\hline DCHWT & 4.3492 \\
DCAHWP & 0.9691 \\
\hline
\end{tabular}

the two methods are applied to Barbara' image Figure 13 aselecting a portion of it of size $(64 \times 64)$, which is marked by the square box in Figure $13 \mathbf{b}$. This small portion corresponds to an image with repetitive variations in intensity (stripes).

The best basis selection by the adaptive wavelet packet for this image is shown in the Figure 14. The subbands /scales in each wavelet packet decomposition level are depicted by square demarcations. The shaded squares are the selected subbands/scales for reconstruction.

The percentage error energy by DCAHWP is about $1 / 5^{\text {th }}$ of that by DCHWT in this case Table 4 . The compressed image by DCHWT is a very crude representation of the image as the stripes are almost not visible (Figure 13c). But the stripes are clearly visible in the compressed image by DCAHWP Figure 13(d).

\section{Conclusions}

An Adaptive Harmonic Wavelet Packet based on Discrete Cosine Transform (DCAHWP) was proposed. The implementation is simple as the DCT coefficients are real. The DCAHWP was achieved by using the entropy criterion realized by comparing the normalized energies of parents and children. The proposed DCAHWP performed better compared to Daubechies-2 based adaptive WP (DBAWP). For a compression factor (CF) of $1 / 8$, for a 1-D signal and speech signal considered, the ratio of the percentage error energy (PEE) by DCAHWP to that by DBAWP was about $1 / 8$ and $1 / 5$, respectively.

Compared to DCT based harmonic wavelet transform (DCHWT), as expected the proposed DCAHWP performed better as the ratio of the percentage error energies by DCAHWP to that by DCHWT better for $1 \mathrm{D}$ and speech, signals respectively were about $1 / 3$ and $1 / 2$, for a compression factor $1 / 8$.

The ratio of PEE for DCAHWP and that for DCHWT for an image $((512 \times 512)$ Barbara) was $2 / 3$ for a CF of $1 / 32$. The compression by DCAHWP is superior in bringing out the edge information (high frequency content) such as eyes, nose and stripes (texture), whereas that by DCHWT results in blurred images. Further for a textural image $((64 \times 64)$, a portion of the Barbara image $)$ this normalized energy ratio between DCAHWP and that for DCHWT it was $1 / 5$ for a CF of $1 / 32$. But in all the cases, the reconstructed signals/images without compression were almost identical to the original.

The results clearly indicate that for signals characterized by abrupt changes or images with rapid variations, the proposed DCAHWP provides significantly better compression performance compared to that of DCHWT. It has been found that the DCAHWP has a significantly better compression performance over the adaptive wavelet packet based on Daubechies-2, DBAWPT.

\section{REFERENCES}

[1] C. Sidney Burrus, R. A. Gopinath and H. T. Guo, "Introduction to Wavelets and Wavelet Transforms: A Primer," Prentice-Hall, Inc., Upper Saddle River, 1998.

[2] P. S. Addison, "The Illustrated Wavelet Transform HandBook,” Institute of Physics Publishing, London, 2002.

[3] R. R. Coifman and M. V. Wickerhauser, "Entropy-Based Algorithms for Best Basis Selection,” IEEE Transactions on Information Theory, Vol. 38, No. 2, March 1992, pp. 713-718.

[4] B. Liu, "Adaptive Harmonic Wavelet Transform with Applications in Vibration Analysis," Journal of Sound and Vibration, Vol. 262, No. 1, 2003, pp. 45-64.

[5] M. Kivanc Mihcak, K. Ramchandran and P. Moulin, "Adaptive Wavelet Packet Image Coding Using an Estimation-Quantization Framework," Proceedings of International Conference on Image Processing, Chicago, USA, Vol. 1, 1998, pp. 92-96.

[6] M. Musaruddin and A. Z. Kouzani, "Embedding Data in Images Using Wavelet Packets,” International Conference IEEE TENCO, Vol. 2, 21-24 November 2004, pp. 120- 123.

[7] D. E. Newland, "Harmonic Wavelet Analysis," Proceedings: Mathematical and Physical Sciences, Vol. 444, No. 1917, 1993, pp. 203-225.

[8] S. V. Narasimhan and M. Harish, "A New Spectral Estimator Based on Discrete Cosine Transform and Modified Group Delay,” Signal Processing, Vol. 86, No. 7, 2006, pp. 1586-1596.

[9] S. V. Narasimhan, M. Harish, A. R. Haripriya and N. Basumallick, "Discrete Cosine Harmonic Wavelet Transform and Its Application to Signal Compression and Spectral Estimation Using Modified Group Delay,” Signal, Image and Video Processing, Vol. 3, No. 1, February 2009, pp. 85-99.

[10] S. V. Narasimhan, A. R. Haripriya and B. K. Shreyamsha Kumar, "Improved Wigner-Ville Distribution Performance Based on Dct/Dft Harmonic Wavelet Transform and Modified Magnitude Group Delay,” Signal Processing, 
Vol. 88, No. 1, 2008, pp. 1-18.

[11] S. V. Narasimhan and A. Adiga, "Shift Invariant Discrete Cosine Harmonic Wavelet Transform and Its Application to Denoising," Proceedings, IEEE International Conference INDICON, Bangalore, 2007.

[12] S. V. Narasimhan and S. Veena, "Signal Processing Prin- ciples and Implementation,” Alpha Science, Harrow, UK and Narosa publishers, New Delhi, India, 2008.

[13] T. Gulzow, T. Ludwig and U. Heute "Spectral-Subtraction Speech Enhancement in Multirate Systems with and without Non-Uniform and Adaptive Bandwidths," Signal Processing, Vol. 83, No. 8, 2003, pp. 1613-1631. 